\title{
8. RAZÓN Y MÍSTICA
}

\section{INTRODUCCIÓN}

Para continuar con nuestra reflexión de teología fundamental, en lo que sigue me gustaría poner en confrontación la razón con sus límites. Lo haré siguiendo ahora a un pensador creyente, y no a uno no creyente, como en el capítulo anterior. En el presente capítulo, acudiré a Leszek Kolakowski, filósofo católico polaco, el cual, sin embargo, fue siempre muy crítico y perspicaz. Por eso, él nos puede ayudar a buscar un equilibrio proporcional o analógico entre la sola razón y la fe pura.

A lo que queda fuera de la razón lo llamamos misterio. Y lo que pertenece al misterio y a su experiencia solemos llamarlo mística. En todo caso, la mística es la vivencia de ese misterio que escapa a lo racional. Como se da en los límites de la razón, todavía alcanza a integrar algo de ella. Es, pues, vivencia de lo misterioso en parte con la razón y en parte con el sentimiento. Esa fusión de horizontes cognoscitivos y vivenciales es lo que también se ha llamado la $f e$, esa creencia que no es opinión cierta justificada, como llamamos a la ciencia, sino otro tipo de opinión, una que está más del lado del sentimiento, del afecto, de la emoción o pasión y, que, sin embargo, nos da cierto conocimiento, el más pleno y plenificante de todos. 
Bertrand Russell tiene un libro que intituló Mysticism and Logic (Mística y lógica), esto es, la razón y la fe, que son los dos tipos de conocimiento que habitan en el hombre. Como diciendo que es la unión de los dos lo que es el mayor desiderátum en el ser humano, como ya lo había asentado Pascal, que lo mejor es unir el espíritu de la geometría y el espíritu de la fineza, pues, quien lograra hacerlo tendría en sus manos el verdadero arte de persuadir. Sea lo que fuere de ello, lo que aquí nos interesa por ahora es esta confrontación con esos dos modos de conocimiento: razón y fe, mística y lógica.

\section{LÓGICA DE LA MÍSTICA Y MÍSTICA DE LA LÓGICA}

Así como puede hablarse de una mística de la lógica (el supremo gozo de guardar la coherencia, de construir sistemas sintácticamente inmaculados), también se puede hablar de una lógica de la mística (aunque la expresión parezca un oxímoron, se refiere a una cierta razonabilidad que siempre cabe en la experiencia mística). Y es que, aun cuando la mística ha sido vista muchas veces como locura y delirio, puede encontrarse en ella una lógica, una racionalidad o razonabilidad como aquella a la que aludía Bergson (1990, pp. 121 y ss.).

Para captar esta lógica o esta razonabilidad (tal vez, racionalidad sea demasiado fuerte) de la mística, tenemos que contextuar su concepto en los límites que la abarcan, esto es, hemos de buscar una definición. De una manera muy amplia, se la puede caracterizar como "una vía o camino de unión o identificación con (o disolución en) lo sagrado, y entender este como un misterio liberador o salvífico" (Cabrera, 2006, p. 11). Se dice que lo sagrado, y no Dios, porque en algunas místicas, como la budista, no hay una divinidad. Y se añade también que no sea una experiencia aislada del misterio, o una 
experiencia extática suelta, ya que tiene que darse en un proceso de marcha hacia lo sagrado (pues, a veces, se llama éxtasis a una alegría natural muy fuerte o a un goce estético, por ejemplo). El proceso suele tener una etapa inicial, una etapa negativa, otra positiva y un después. La etapa inicial es dolorosa, de mucha abnegación; la negativa es de cierto desconocimiento, de lenguaje negativo; la positiva es de unión con lo sagrado, y el después de esa unión es el sentirse transformado para vivir con los demás en caridad (cristianismo) o en compasión (budismo) (Cabrera, 2006, p. 19).

Las experiencias místicas tienen mucho de común, pero también mucho de diverso. Podría decirse que el camino es semejante, pero el sentimiento muy distinto. Y la experiencia mística es un fenómeno humano, que merece ser estudiado, incluso si se considera que nada objetivo le responde, esto es, que no hay algo sagrado, o un Dios. Sin embargo, la existencia de los místicos es lo que más da testimonio de la religión, y lo que acerca más a pensar que tiene alguna validez. Sigue siendo cierto lo que decía Bergson, que los místicos o, más propiamente, los santos son los que dan mayor validación de la religión o del hecho religioso.

Mas pasemos a la religión y la razón, o de cómo es vista la fe religiosa por los racionalistas. La ven como algo falso, pero, según parece, no hay privilegio para el racionalista por encima del creyente. Ambos están igual, tienen la misma imposibilidad de demostrar sus fundamentos últimos.

Desde hace tiempo, se ha tenido la impresión de que el teísmo y el ateísmo (o agnosticismo) están en paridad de razón (pari ratione), es decir que están empatados. Ninguno tiene de suyo la preeminencia o el gane, y ninguno tiene a priori la carga de la prueba u onus probandi. Ni el teísmo es a priori falso ni el ateísmo a priori verdadero, aunque lo parezca prima facie. 
Se ha argumentado que los argumentos racionales del teísmo se pueden bloquear, y que también los argumentos contrarios a ellos se pueden bloquear en algún punto. $\mathrm{O}$, por lo menos, no son concluyentes. Lo malo es que si no se puede demostrar racionalmente la existencia de Dios, tampoco se puede demostrar racionalmente su inexistencia, y ni siquiera el que tengamos que ser racionalistas; por lo menos tendría que ser con otro tipo de argumentos (pragmáticos o pragmatistas, por ejemplo, pero también validarían la creencia en Dios).

En otras palabras, el teísmo no se ha podido demostrar con los argumentos que se han dado. Quizá no haya argumento que lo haga. Pero tampoco hay argumento que demuestre la no existencia de Dios. Porque, en primer lugar, no hay argumento que demuestra la validez de la razón, su verdad completa. Eso llevaría a tener que aceptar una verdad y una mente absolutas.

\section{RAZÓN Y FE}

Leszek Kolakowski (1988, pp. 83-91) aporta esta argumentación para hacer ver que, si no hay Dios, no hay verdad posible. Es decir, solo 110 tiene sentido hablar de verdad si se supone una Mente absoluta. Fue lo que vio Descartes, cuando apoyó la verdad en un Dios que no miente. Tomará la verdad en el sentido del primer Husserl, esto es, trascendental (verdadero es lo que de verdad ocurre, a pesar de que no lo sepamos o no lo verifiquemos), y no en el sentido de Heidegger.

Las reglas, criterios y definiciones de la verdad no se pueden inferir de los hechos, que están, precisamente, en tela de juicio. Por lo tanto, o caemos en una regresión infinita, o efectuamos una decisión discrecional (Kolakowski, 1988, pp. 84-85). Si definimos la verdad como el cientificista, mediante sus criterios de eficacia, estamos efectuando esa decisión arbitraria, es un acto de fe: 
Podemos definir la verdad, ciertamente, mediante la referencia a los criterios de eficacia; tal definición no es autocontradictoria y no lleva a una retrogresión infinita; no obstante, es arbitraria; aceptarla requiere un acto de fe y, por tanto, el principio credo ut intelligam opera sobre todo el campo del conocimiento (1988, p. 85).

Es curioso encontrar que el principio de la teología cristiana, creer para entender, está en la base de todo conocimiento, incluso el científico (esto es algo que ya desde antes decía Popper y, antes que él, Peirce. Este último decía que no podemos demostrar que tenemos que ser racionales, pues, tendríamos que hacerlo con la razón, lo cual es un círculo vicioso, petición de principio, o tendríamos que hacerlo con algo distinto de la razón, la fe o la voluntad, por ejemplo, y eso sería peor. Popper añadía que no podemos demostrar que la ciencia es lo mejor para nosotros, lo aceptamos por una especie de acto de fe).

Ciertamente, esto no afecta a la ciencia reciente, pues, ella es consciente de que no busca la Verdad, sino una justificabilidad o confiabilidad que le resulta suficiente.

Kolakowski recuerda que el primer Husserl quiso rebatir esto diciendo que cuando el escéptico dice "Nada es verdad", ya al menos hay algo verdadero, que no hay verdad, y se contradice o autorrefuta. Pero Kolakowski añade que el escéptico rechaza que "El sol brilla" equivalga a "Es verdad que el sol brilla". Husserl tuvo que acudir al Ego trascendental, que es una especie de Mente absoluta (1988, pp. 87-88). Es verdad que el conocimiento de Dios no nos dice dónde está la verdad, o qué cosas son verdaderas, pero nos dice que algo, por lo menos, es verdadero. Incluso plantear verdades parciales implica sostener una verdad absoluta. "Así, sin la verdad que todo lo abarca, no hay verdad fragmentaria; y la verdad que todo lo abarca presupone una inteligencia infinita y omnisciente. Solo ella puede 
cumplir lo que Husserl esperaba de un Ego trascendental" (p. 90). Pero en una mente absoluta no hay distinción entre sujeto y objeto, pues, es la transparencia plena frente a sí mismo. Por eso, la noción de verdad no tiene sentido para Él, y vuelve a desaparecer. Pero se mantiene la intuición cartesiana de que la verdad solo es posible si se refiere a una verdad que todo lo abarca, una mente infinita.

Con todo, esto no vale como prueba de la existencia de Dios, solo de la no existencia de la verdad sin Dios. Explica Kolakowski:

Sin embargo, el argumento no tiene nada que ver con las pruebas de la realidad de Dios: no puede reforzar ni debilitar ninguno de los argumentos existentes. Su objetivo no es mostrar que Dios existe en efecto, sino exponer el dilema al que parecemos enfrentados cuando abordamos la cuestión de la verdad y de la posibilidad misma de la epistemología: o Dios o un nihilismo cognoscitivo, no hay término medio (p. 91).

Tal es la fuerte situación en la que nos encontramos. El teísmo no ha podido demostrar racionalmente la existencia de Dios, pero el ateísmo racionalista no puede demostrar ni siquiera la razón misma.

El reduccionista (ateo o agnóstico) está atrincherado en sus propios criterios, ha hecho un perímetro de defensa, el cual es tramposamente inmune a toda objeción. Ya sea lógicamente, pues, son presuposiciones tan básicas que no puede poner en duda (pero que, si se ponen en duda, muestran su fragilidad, como el hecho de que ahora, si se cuestiona la validez de la ciencia, no se sabe cómo apuntalarla más que con argumentos pragmáticos). Ya sea sociológicamente, pues, al que niegue su mecanismo de reducción lo llamará alienado, revisionista, defensivo, etcétera. Y rechazará la experiencia religiosa como sueño o como locura, como ilusión o como delirio. 
Frente a eso, el teísta suele escudarse en que la fe no se puede someter a la razón, que es un sentimiento, etcétera. Dios no es una hipótesis científica, la ciencia solo tiene validez por su utilidad, no se puede poner a discusión, etcétera.

De lo que no se dan cuenta es que son dos formas de vida, con sus juegos de lenguaje elaborados desde los presupuestos de cada una. Los presupuestos del racionalismo dan como resultado los filtros epistemológicos para los que fueron hechos. Lo que no pase esos filtros no es razonable.

Pero la percepción del mundo en términos de un orden bajo la dirección divina es igualmente coherente y proporciona al creyente[,] una comprensión por la que el racionalista rehúsa interesarse, sencillamente. La certeza del creyente, en efecto, no puede expresarse en un lenguaje que cumpla los requisitos del discurso científico y, de hecho, el lenguaje de lo Sagrado no es una parte ni una extensión del lenguaje cotidiano; es inteligible dentro de la vida de lo Sagrado, en los actos del culto (p. 213).

Esto implica que hay diversos criterios de verdad en el racionalismo y en el teísmo. No es la idea de la doble verdad, de los averroístas latinos, sino, simplemente, la diversa aplicación (analógica) de los criterios de verdad que tenemos para hacer razonable algo. Demostrar la fe es autocontradictorio, no se trata de eso; se trata de aportar argumentos que hagan creíble, esto es, razonable o aceptable por no ser irracional el contenido de la fe.

En efecto, si se concibe la continuidad como un criterio de verdad en el sentido normal de la palabra, las afirmaciones de los cuerpos religiosos, según las cuales ellos son portadores de verdad, tienen que parecer absurdas. Pero no lo parecen en absoluto si se tiene en cuenta la idea de validez que es específica del dominio de lo 
Sagrado. Puesto que, como he tratado de mostrar, la religión no es un conjunto de proposiciones, sino una forma de vida en la que entendimiento, creencia y compromiso convergen en un acto único (algo que se expresa con torpeza en términos doctrinales) y puesto que las personas entran en esta forma de vida como consecuencia de su iniciación real al culto comunitario, parece natural que la verdad religiosa se preserve y transmita en la continuidad de la experiencia colectiva (p. 218).

Con esto se elimina la posibilidad, demasiado simple, de acabar con la religión bloqueando los argumentos teístas, pues, se trata de algo más holístico (parecido al holismo epistemológico de Duhem y Quine). Hay que tomar en cuenta la práctica religiosa completa, como forma de vida, dentro de la cual cabe muy bien la epistemología de las virtudes, ya que las virtudes epistémicas se dan en un contexto de vida, en una comunidad, y, de acuerdo con su dinamismo, nacen y se desarrollan. Es decir, no hay que separar la razón y la fe, sino unirlas, concordarlas, hacerlas que trabajen la una para la otra (en una especie de coincidentia oppositorum).

\section{HUNDIR LAS RAÍCES EN EL MITO}

Para ir más a fondo en las relaciones entre la fe y la razón, es el mismo Kolakowski quien nos guía. Sostiene que ambas hunden sus raíces en el mito. Dice que la fuente de la religión es el mito. Entendiendo mito en el buen sentido, como aquello que es simbólico y da sentido para vivir. En un interesante libro intitulado La presencia del mito, nos hace ver que el mito está presente, incluso en la actualidad. El mito se encuentra en la cultura y ubica al hombre en el cosmos. Le señala la contingencia de la naturaleza; le habla a pesar de la indiferencia y el mutismo del mundo; lo acompaña en la existencia, recalcándole su conocimiento y su libertad. En efecto, el mito está 
en el problema del conocimiento, y nos ayuda a confiar en la razón, ya que nuestra confianza en la razón y en la ciencia está basada en un mito, un mito que está más allá de la ciencia misma y de sus alcances (1990, pp. 20 y ss). Incluso se halla en la lógica y, sobre todo, en el mundo de los valores. La axiología del hombre (al igual que la epistemología, pero todavía más) tiene su fundamento en el mito.

Pero, de manera especial, el mito se encuentra en las raíces de la religión. Así como Kolakowski sostiene que la creencia religiosa es indemostrable (al igual que lo son los principios de la razón, según ya señalaba Pascal), así también el filósofo polaco nos indica que la religión (al igual que los productos de la razón) hunden de manera lejana sus raíces en el mito. El mito es lo que da sentido, la fuente de significado para el hombre en la vida. Asevera:

El mito da sentido al mundo, pero el movimiento de nuestra intención, referida al contenido del mito, no es comprender, al menos no predominantemente comprender. Ella es el acto simultáneo de entrega triple, que los cristianos han repartido en la Trinidad que denominaron virtudes teologales (p. 52).

Tales virtudes son nada menos que la fe, la esperanza y la caridad.

La tesis de Kolakowski es que la fe, la esperanza y la caridad solo pueden apoyarse en la esfera mítica, y eso hace que las distingamos de sus homónimas no sacras, sino profanas, las cuales no llegan a la energía que aquellas tienen. Efectivamente, confiamos en un reloj, que no ha fallado hasta el momento presente; pero la confianza en una persona es muy diferente. Es una confianza total y sin justificación racional plena. En la fe, en la confianza, aceptamos a una persona por su constitución mítica, por su simbolismo o simbolicidad, porque representa para nosotros una seguridad, pero no como las regularidades de la naturaleza, sino incluso desde su libertad. 
En toda fe está coimplicada la cualidad del mito, pero también a la inversa: en toda relación con el mito hay coimplicado un acto de fe, aunque aquel no es una realidad personal. Volviéndonos al mito, como una instancia mediante la que se hace comprensible la experiencia, lo dotamos con nuestra confianza, pero la fuente de esta confianza no la vemos en nosotros, sino en aquello a lo que está dirigida (p. 53).

La misma decisión que está detrás de la fe acompaña a la esperanza. No se queda en mera probabilidad que se espera de ciertos acontecimientos, sino que es una guía en el camino para llegar a las realidades que se desea encontrar. La esperanza es el deseo de intercambio de existencias con otro, deseo que se desea que no acabe y, sin embargo, se busca su término. Ese deseo es también sin fundamento racional; solo se basa en una actitud mítica. Se da en medio de la fragilidad, de la limitación, pero alcanza autosuficiencia. "Por tanto, la esperanza está contenida, igual que la fe, en cada uno de mis giros hacia el mundo mítico, e, igualmente, el objeto de la esperanza se transmite siempre en el ámbito del mito" (p. 54).

De manera parecida ocurre con la caridad, con el amor. Igual que en el caso de las dos cualidades anteriores, el sentido mítico del amor está determinado doblemente: en el amor hay coimplicada una intención referida a la realidad constituida míticamente, $y$, a la inversa, toda energía dirigida al mundo mítico conlleva un ímpetu erótico (p. 54).

Kolakowski encuentra en el amor una totalidad de anhelo, una vivencia de la originalidad, una infalibilidad, una ausencia de pretensiones y atribuciones, un intento de suspender el tiempo y una originalidad del todo frente a las partes. 
Es anhelo de unión, de superación de la distancia. Es disgusto por la separación, esperanza de unificación y sacrificio hasta la disolución en el amado. Esta experiencia coincide con la de los místicos, los cuales solo han encontrado esta manera de expresarla. Esto es lo más interno al mito, en el que este encuentra su realización definitiva. Tal vez, solo de manera mítica se puede narrar la experiencia del amor. Además, es vivencia del origen, de lo que estaba al principio. Siempre se ve como retorno a algo muy íntimo y profundo, como esperanza de encuentro con lo original. Por eso, se ve como el Paraíso, el cielo. La gloria eterna solo podría ser el cumplimiento del amor, como un comienzo sin fin. El amor y la satisfacción se excluyen, el amor es eterno comienzo, eterno retorno al origen. Asimismo, el amor contiene una infalibilidad, una certeza absoluta, no racional (no como el amor intelectual a Dios, de Spinoza), sino de índole mítica, sin fundamento en la razón. La empatía entre dos existencias solo tiene su base en el mito, en un ámbito simbólico. En ese sentido, en el amor hay ausencia de pretensiones; no hay interés, ni cálculo, ni obligación, es completamente libre. Es donde se comprende el significado de la gracia, de lo que se da gratuitamente. Hay también en el amor una suspensión del tiempo. Es la espera que se actualiza, es el deseo que se realiza, es un tiempo sin tiempo, es el momento eterno. Por eso, supera al tiempo histórico. Es lo que se trata de atrapar en el rito, en los rituales religiosos. Es el deseo de repetir, la repetición de la identidad, hasta que se haga indefectible. Así es la relación amorosa y el éxtasis místico, por ello, llevan a cierto nihilismo, que desata de lo temporal, de lo terreno, de lo real.

El amor escapa al recuerdo y a la previsión, al pasado y al futuro, y se erige como presente. Desaparece toda mediación, y solo queda el contacto directo. Los místicos la llamaron realización de la eternidad en el tiempo. No pertenece al tiempo real, sino al tiempo mítico, por denominarlo de alguna manera. Finalmente, el amor conlleva una 
originalidad del todo en relación con las partes. Hay un enlace holístico, que supera la fragmentación e instaura la totalidad, incluso pone como origen el todo. "No avanza, por tanto, de las partes al todo, sino que transmite la plenitud del todo a cada parte singular. Tanto en la pasión teopática como en la corporal descubrimos de nuevo la misma estructura" (p. 57). En el caso del amor de Dios, se da la adoración de Dios en totalidad, sin fundamento racional, sino mítico o místico. Por consiguiente, Kolakowski ve con tan malos ojos las teodiceas, porque no alcanzan a expresar la riqueza de esa experiencia y, como basadas en la razón, tienen la mala fe de querer disculpar a Dios del mal que hay en el mundo, mientras que el amor a Dios no intenta disculparlo de nada. Y, en el caso del amor a otro ser humano, se lo ama en su totalidad, no por este o aquel aspecto, sino que, al revés, el amor a la totalidad hace que se amen también en él tal o cual aspecto. El amor todo lo acepta y todo lo disculpa. Es un valor mítico, la razón no alcanza para hacerlo.

\section{VISIÓN DESDE LA HERMENÉUTICA ANALÓGICA}

Al trasluz de una hermenéutica analógica se pueden aceptar algunos puntos de vista de Kolakowski, aun con ciertas modificaciones. Está visto que la religión no se puede demostrar racionalmente. Las pruebas que se han aducido a su favor tienen defectos y no alcanzan a dar ese examen. Eso es bueno, pues, si concluyeran apodícticamente no habría fe, sino demostración. La fe perdería su sentido. Pero tampoco han funcionado las pruebas en contra de la existencia de Dios. $\mathrm{O}$ bien no concluyen y se pueden bloquear en algún punto, o bien suponen ellas mismas lo que demandan, pues, acaban por carecer de bases racionales, en cuanto la razón misma, como lo han mostrado Peirce y Popper, no puede demostrar su fuerza y encierra una gran petición de principio, que afecta a sus pretensiones de acabar con 
el contenido de la religión. Mas por la misma imposibilidad de demostrar la no existencia de Dios esta tiene razonabilidad, una fuerza tópica o dialéctica o de argumentación por lo menos retórica.

En una hermenéutica analógica, la religión no es demostrable racionalmente, pero tiene por lo menos razonabilidad, es razonable, argumentable y defendible con argumentos dialécticos, tópicos o, por lo menos, retóricos (Beuchot, 2009d). Pero será más grande su apoyo en la experiencia, como pide el propio Kolakowski. Es el argumento de los místicos, en seguimiento del gran Bergson.

O pueden funcionar argumentos pragmáticos, como el de Pascal, de la apuesta en la que nos lanzamos a la creencia en Dios y, si no hay Dios, no perdemos nada, pero si hay, lo ganamos todo. O como el de Peirce, de una abducción para la que Dios es la mejor hipótesis para explicar el universo. O como la de James, de que Dios le dio un sentido para vivir, para superar las intensas depresiones que casi lo conducen al suicidio.

En todo caso, en una hermenéutica analógica, es más un Dios de sentido que de referencia. Más de dar valor para vivir que de depender de las pruebas que atestigüen su existencia. Se muestra trabajando, aportando esa carga de sentido que el hombre necesita para vivir, más allá de la referencia que Él necesita para existir.

Kolakowski descree de las pruebas de la existencia de Dios, desconfía de las teodiceas, pero confía en los místicos, en esos seres extraordinarios que se acercan a Dios, que mantienen relación estrecha con Él y que nos dan su testimonio. Que alimentan nuestra frágil fe, y nos muestran mucho (y nos dicen un poco) de su secreto. En esto coincide con Bergson, en que la única prueba de la dimensión religiosa son los místicos. 
Aquí nos resuena, también, el dictum de Wittgenstein, de que lo místico no se puede decir, solo se puede mostrar. Pero nos parece excesivo, y a nuestro favor está toda una tradición mística que trata de hablar, de decir algo, de no quedarse solo en el mostrar. Si el mostrar y el no decir son de la teología negativa, el decir, además de mostrar, es de una teología positiva, pero a medias, una teología analógica. Místicos como Eckhart y san Juan de la Cruz, en la línea de san Agustín y santo Tomás de Aquino, usaron el lenguaje analógico para decir aquello que solo podrían mostrar, resistiéndose a solo mostrar, esforzándose por decir, aunque sabiendo que únicamente lo podrían decir a medias, solo balbucir, en parábolas, metáforas y poesía, que todo eso es lenguaje analógico.

Es a lo que conduce, y es donde se detiene, sabedora de sus límites, una hermenéutica analógica, una racionalidad analógica, con su lenguaje analógico, en una teología casi negativa, pero no lo es, porque trata de ser un poco positiva, una teología analógica. En ella predomina la mística, la experiencia, pero también hay algo de teología, precisamente una que se alimenta de lo que le dan los santos.

\section{CONCLUSIÓN}

Kolakowski huye de la mera equivocidad a la que se arriesga la teología negativa, la cual acaba por no decir nada, y trata de evitar la pretensión de univocidad de la teología positiva, y busca una teología intermedia, la teología analógica. Por eso, desecha la teodicea, y quizás está en lo correcto, pero también desconfía de la teología, pero aquí hay que matizar, hay que distinguir. La que parece poner en entredicho es la teología positiva unívoca, por ello, proponemos una teología positiva analógica, que es casi una teología negativa, pero sin el peligro de recluirse en la equivocidad. Trata de decir sin decir, trata de mostrar diciendo, casi trata de decir el mostrar. 